\title{
ATUAÇÃO DO PROFISSIONAL ENFERMEIRO NO CUIDADO À PESSOA COM DIABETES MELLITUS: REFLEXÕES À LUZ DA TEORIA DO CUIDADO HUMANO
}

\author{
PERFORMANCE OF THE NURSE PROFESSIONAL IN CARE FOR PEOPLE WITH \\ DIABETES MELLITUS: REFLECTIONS IN THE LIGHT OF THE THEORY OF \\ HUMAN CARE
}

\author{
Lara Pereira Leite Alencar, João Cruz Neto, Gledson Micael da Silva Leite, Héryka Laura Calú \\ Alves, Grayce Alencar Albuquerque
}

Universidade Regional do Cariri

Abstract

The article aims to identify in the scientific literature actions to be developed by the nurse professional in the care of people with diabetes mellitus in the light of the Human Care Theory. This is a narrative, descriptive review, carried out through access to studies indexed in Latin American and Caribbean Literature in Health Sciences (LILACS), Nursing Database (BDENF) and PubMed. Forms of distribution and categorized 27 articles related to the theme according to the ten basic factors of the Theory of Human Care. It was identified that nursing actions towards people with diabetes, such as recognition and empathy in the face of suffering, appreciation of spirituality and construction of a physical space and communication, still occur in a deficient way; actions such as stimulation of the individual's expressions, identification of his feelings and behavior and the practice of welcoming are worked in a more profound and horizontal way; as well as, the search for more in-depth knowledge about diabetes by professionals and the exercise of health education are considered essential for assistance. It has to be said that the contemplation of the factors of the Human Care Theory as guiding the professional conduct of nurses to people with diabetes can directly imply the provision of holistic and quality care.

Keywords: Nursing; Diabetes Mellitus; Primary Health Care; Nursing Care; Nursing Theory.
Resumo

O artigo tem como objetivo identificar na literatura científica ações a serem desenvolvidas pelo profissional enfermeiro no cuidado a pessoas com diabetes mellitus à luz da Teoria do Cuidado Humano. Trata-se de uma revisão narrativa, descritiva, realizada por meio do acesso aos estudos indexados na Literatura Latinoamericana e do Caribe em Ciências da Saúde (LILACS), Base de Dados de Enfermagem (BDENF) e PubMed. Foram analisados e categorizados 27 artigos afins ao tema de acordo os com dez fatores básicos da Teoria do Cuidado Humano. Foi identificado que as ações da enfermagem frente às pessoas com diabetes, tais como reconhecimento e empatia frente ao sofrimento, valorização da espiritualidade e construção de um espaço físico e de comunicação acontecem ainda de forma deficiente; ações como estímulo das expressões do indivíduo, identificação de seus sentimentos e comportamentos e prática do acolhimento precisam ser trabalhadas de forma mais profunda e horizontal; bem como, a busca de conhecimentos mais aprofundados sobre diabetes pelos profissionais e o exercício da educação em saúde são tidas como imprescindíveis para assistência. Tem-se que a contemplação dos fatores da Teoria do Cuidado Humano como norteadora da conduta profissional do enfermeiro às pessoas com diabetes pode implicar diretamente na prestação de um cuidado holístico e de qualidade

Palavras-chave: Enfermagem; Diabetes Mellitus; Atenção Primária à Saúde; Cuidados de Enfermagem; Teoria de Enfermagem. 


\section{Introdução}

No Brasil, o diabetes mellitus (DM) já acomete mais de $7,5 \%$ da população, sendo esta, principalmente, pessoas acima dos 40 anos de idade e com comorbidades, como a hipertensão arterial e hipercolesterolemia ${ }^{1}$. Ainda, o elevado número de casos de DM está atribuído a algumas condições, como o sedentarismo, obesidade, envelhecimento populacional e as alterações no estilo de vida ${ }^{2}$.

O diabetes acarreta complicações crônicas e agudas graves quando não tratado adequadamente $^{3}$. Por ser uma doença de caráter crônico, torna-se imprescindível o acompanhamento contínuo durante toda a vida do indivíduo, o que justifica a necessidade da assistência de uma equipe multiprofissional para o controle dos agravos ${ }^{4}$.

O profissional enfermeiro, no contexto de controle desta enfermidade, assume importância, pois desenvolve a função de prestar assistência contínua e ampla para o indivíduo, família e comunidade. $\mathrm{Na}$ consulta de enfermagem para pessoas com DM, deve-se identificar fatores de riscos correlacionados à doença e realizar atividades de educação em saúde, objetivando a prevenção das complicações da doença ${ }^{5}$.

Para tanto, o cuidado de enfermagem deve ser direcionado de forma individualizada e sistematizada. Este cuidado deve seguir protocolos instituídos para uniformizar as ações no cuidado às pessoas com $\mathrm{DM}^{6} \mathrm{e}$ desta forma, compete ao enfermeiro ajudar a pessoa com DM na compreensão da necessidade de assumir alterações no estilo de vida e aderir ao tratamento, o que vai contribuir para o controle glicêmico ${ }^{7}$. Para alcance deste objetivo, as Teorias de Enfermagem fundamentam o cuidado, com conhecimentos próprios, a fim de melhorar a qualidade da atenção direcionada às pessoas em diferentes campos de conhecimento ${ }^{7}$.

Corroborando com tais assertivas, a Teoria do Cuidado Humano, elaborada entre por Jean Watson (1975-1979) descreve 10 fatores básicos para a realização do cuidado, denominados de fatores cuidativos que são: 1 formação de sistema de valores humanistaaltruísta; 2 - a instilação da fé-esperança; 3 - o cultivo da sensibilidade do próprio self e das demais pessoas; 4 - o desenvolvimento de uma relação de ajuda e confiança; 5- a promoção e aceitação da expressão de sentimentos positivos e negativos; 6 - o uso sistemático de um processo criativo de solução de problemas; 7 - a promoção do ensino-aprendizagem transpessoal; 8 - a provisão de um ambiente de apoio, proteção e/ou neutralização mental, física e espiritual; 9 assistência com gratificação das necessidades humanas e 10 - a permissão de forças existenciais-fenomenológicas-espirituais ${ }^{8,9}$. Desta forma, acredita-se que a aplicação de uma Teoria de Enfermagem pode contribuir para um cuidado de enfermagem holístico e centrado na individualidade de cada pessoa, fortalecer o vínculo, a adesão à terapêutica proposta, bem como, reduzir danos e sequelas de uma patologia, nesse a DM.

Embora importante, os cuidados de enfermagem prestados a pessoa com DM podem ser realizados sem aproximação com aportes teóricos orientadores, então, faz-se necessário conhecer quais cuidados estão sendo realizados, quais as devidas orientações para sua realização e como devem ser aplicados a partir das diretrizes teóricas eleitas, a exemplo da Teoria do Cuidado Humano. Assim, levando-se em consideração essas condições e a necessidade de um cuidado pleno ao indivíduo com DM, esse artigo objetivou identificar na literatura científica, ações a serem desenvolvidas pelo profissional enfermeiro no cuidado a pessoa com diabetes mellitus à luz da Teoria do Cuidado Humano.

\section{Metodologia}

Para a elaboração deste trabalho, utilizou-se uma revisão narrativa, de cunho descritivo ${ }^{10}$. Foram analisados artigos científicos sobre cuidado de enfermagem frente ao diabetes mellitus, sob a ótica da Teoria do Cuidado Humano. Foram selecionados artigos completos, disponíveis online e escritos em português, espanhol e inglês, sem determinação temporal. Foi realizada busca nas bases de dados da Literatura Latino-americana e do Caribe em Ciências da Saúde (LILACS), Base de Dados de Enfermagem (BDENF) e portal PubMed.

Utilizaram-se os descritores: enfermagem, cuidados de enfermagem e diabetes mellitus e seus Medical Subject Headings (MeSH) nursing, nursing care e diabetes mellitus, juntamente com o operador booleano $A N D$. Foram encontrados nas bases de dados 68 artigos, com amostra final de 27 que respondiam ao objetivo desta revisão, sendo que 41 estudos foram excluídos pelo seu conteúdo não contemplar especificamente os cuidados de enfermagem a pessoa com DM.

Após definição da amostra final, os cuidados de enfermagem identificados nos estudos foram avaliados e categorizados 
levando-se em consideração os 10 fatores da Teoria do Cuidado de Jean Watson ${ }^{9}$ e analisados conforme à literatura pertinente.

Por se tratar de uma revisão narrativa, este estudo não precisou ser submetido e apreciado por Comitê de Ética em Pesquisa.

\section{Resultados e Discussão}

Fatores de Cuidados em Enfermagem ao com Diabetes Mellitus

A promoção do cuidado se dá por meio de uma interligação entre o enfermeiro e o individuo que necessita de cuidados, na qual o profissional, embasado na sua formação, será capaz de dar suporte ao cliente, no que diz respeito à promoção, prevenção e reabilitação da saúde, devendo levar em consideração, durante a prestação dos cuidados, a materialização dos 10 fatores inerentes à Teoria do Cuidado Humano.

O cuidado de enfermagem pode ser realizado nos mais diversos ambientes, sejam em escolas, hospitais, domicílios e afins. Dessa forma, é primordial a capacidade de atuar e prestar assistência de forma integral ao indivíduo em todos os âmbitos que o compõem. Dentre esses, na atenção primária, que desempenha um papel essencial na organização do sistema de saúde, e é considerada a porta de entrada para a assistência.

Neste setor, o enfermeiro atende as mais diversas faixas etárias, como também uma diversidade de morbidades, principalmente condições crônicas, como é o caso do DM, devendo, portanto, estar atento ao desenvolvimento de uma prestação de cuidado holística, de forma a garantir redução das complicações dessa doença e o protagonismo da pessoa em sua reabilitação.

Nesse sentido, torna-se importante um cuidado embasado cientificamente, que se guie por parâmetros e diretrizes de forma a potencializar a assistência prestada, no sentido de atender às demandas do indivíduo e reduzir os riscos de agravos. Conhecer como deve ocorrer a prestação de uma assistência de enfermagem satisfatória a pessoa com DM, implica em adequá-las à excelência. Corroborando com este pensamento, o cuidado de enfermagem a ser prestado a pessoa com DM pelo enfermeiro é analisado frente à aplicabilidade dos 10 fatores da Teoria do Cuidado Humano (Quadro 1).

<Inserir Quadro 1>
Fator 1 - Formação de sistema de valores humanista-altruísta

Os valores humanista-altruístas emergem do comprometimento e da satisfação pessoal em auxiliar outra pessoa. O enfermeiro que expressa o comportamento altruísta em relação a si e aos outros, possui melhores condições de vivenciar o cuidado humano e essa condição reflete os valores pessoais de quem cuida e de quem é cuidado, e mostra o que a pessoa é na sua mais íntima essência ${ }^{11}$.

Neste sentido, o enfermeiro deve ser capaz de compreender os sentimentos e razões de outra pessoa, além de poder prever quais os comportamentos e pensamentos adotados por esta, por meio da simulação da perspectiva experienciada pelo outro.

Torna-se imprescindível que o enfermeiro se coloque no lugar da pessoa e a partir disso, consiga atender as necessidades deste, sem levar em conta apenas os fatores físicos que o compõem, vendo-o sempre de forma integral. Trata-se de mudar o foco de ações instrumentais e de procedimentos para algo mais amplo. Ainda, o profissional que é capaz de transformar interações com o indivíduo, expande seu campo de ação, possibilitando atenção mais ampla para quem necessita de cuidado ${ }^{9}$.

No entanto, o que se observa na assistência frente ao indivíduo com DM ainda é a carência desse componente cognitivo nas relações profissionais. Em uma pesquisa realizada com pessoas com DM e profissionais de saúde, dentre eles enfermeiros, revelou-se que o reconhecimento e empatia frente ao sofrimento gerado pelo adoecimento ainda é incipiente, reproduzindo-se o enfoque biomédico com maior ênfase, em que os cuidados relacionados ao corpo físico, a disposição para mudança, os hábitos de vida e a avaliação quantitativa de indicadores são preponderantes na assistência ${ }^{12}$. Assim, fica nítido na assistência de enfermagem atual a pessoa com DM, que este fator da Teoria do Cuidado Humano ainda não é preponderante na atenção dispensada.

\section{Fator 2 - A promoção da fé-esperança}

O enfermeiro e outros profissionais da saúde não podem ignorar o importante papel da fé e esperança no cuidado e no processo de cura, uma vez que se sabe que a fé-esperança pode ajudar o cliente a aceitar informações e se engajar em atitudes de mudança na busca de comportamentos saudáveis ${ }^{8}$. 
É fundamental que o cliente seja visto como ser que possui crenças, valores e princípios. É necessário considerá-lo como alguém importante para o universo e para aqueles que o amam. Assim, este deve ter suas crenças e doutrinas respeitadas e estimuladas pelo enfermeiro, pois frequentemente a fé e esperança tornam-se alicerce para a aceitação e adesão ao tratamento proposto ${ }^{9}$.

É indispensável que haja o enfoque na espiritualidade, esperança e otimismo na assistência aos indivíduos com diabetes, estas devem ser aceitas e estimuladas pelos profissionais de saúde ${ }^{13}$. Ademais, a espiritualidade torna-se alicerce para que 0 indivíduo consiga enfrentar a doença.

Em virtude do DM ser uma doença que acarreta medo, estresse e incerteza, as práticas religiosas podem vir a ser um alicerce para o enfrentamento $^{14}$. De fato, se observa que a religiosidade tende a elevar-se diante eventos de vida negativos, incluindo o adoecimento ${ }^{15}$. Isto ocorre porque a conexão com a religião pode ser uma fonte de alívio ou desconforto, dependendo de como a pessoa se reporta a ela ${ }^{16}$. Neste sentido, é importante que o profissional de enfermagem reconheça e estimule a espiritualidade dos indivíduos como forma de reduzir possíveis desconfortos, potencializar a adesão ao tratamento e promover melhorarias à qualidade de vida.

Fator 3 - O cultivo da sensibilidade do próprio "eu" e a das demais pessoas

O cultivo da sensibilidade do "eu" e das demais pessoas expõe a carência que as relações de cuidado têm em relação à emoção e também acaba por contribuir para a construção de relações afetivas, amorosas e humanizadas ${ }^{11}$.

Torna-se assim, essencial, que haja o fortalecimento do vínculo entre profissional e usuário nas práticas de atenção à saúde, fomentando o atendimento humanizado. 0 mesmo configura-se pela escuta sensível, que leva em conta todos os anseios e angústias do indivíduo, tanto no acolhimento, como em todo o acompanhamento, sendo a pratica embasada na ajuda mútua e respeito recíproco ${ }^{17}$.

Ainda, as soluções para os problemas de saúde podem ser identificadas por meio de dessa escuta sensível e empática para com a pessoa. Quando o profissional de saúde assume esse papel de ouvinte, os laços entre ele e o usuário do sistema torna-se mais estreito, sendo o profissional visto não somente como um enfermeiro, mas sim como alguém que o indivíduo pode confiar seus anseios ${ }^{17}$.

$\mathrm{Na}$ assistência de enfermagem é preciso que haja humanização, que o enfermeiro extrapole as barreiras de um modelo segmentado e meramente curativo, atuando para além da estrutura física do indivíduo, enfatizando também seus outros aspectos, como os psicológicos e emocionais ${ }^{18}$. Para tanto, faz-se necessário que o enfermeiro possa ser sensível à identificação dos sentimentos e comportamentos que dificultam a aceitação da condição crônica de saúde pela pessoa e, consequentemente, a adoção de hábitos saudáveis que permitam lidar com as limitações decorrentes da enfermidade ${ }^{19}$.

Fator 4 - O desenvolvimento de uma relação de ajuda-confiança

O estabelecimento de uma relação de ajuda-confiança é um modo de originar vínculo entre os participantes do processo de cuidado. É primordial que o enfermeiro diante do seu cliente, demonstre atenção e empatia, uma vez que é por meio dessas ações que poder-se-á desenvolver uma relação de confiança e respeito 9 .

Há ainda que por meio de um elo, o enfermeiro conhecerá todos os fatores que estão relacionados ao processo saúde-doença, os determinantes e condicionantes para tal e assim, poderá traçar um plano de cuidados que se enquadre na realidade do cliente ${ }^{20}$.

É indispensável que o profissional de saúde saiba ouvir o usuário, no intuito de aferir aspectos que possam estar influenciando no tratamento da doença, uma vez que a não utilização da escuta qualificada, poderá influenciar na terapêutica do indivíduo com DM. A interação entre profissionais de saúde e usuários configura-se como algo complexo de se estabelecer, sendo a capacitação e motivação por parte dos profissionais, ferramentas primordiais para que haja a mudança desse quadro ${ }^{21}$.

O relacionamento horizontalizado é visto por meio da liberdade de expressão por parte dos usuários, como também o compartilhamento de experiências, em uma relação amigável e empática entre o usuário e profissional, o que facilita o enfrentamento da condição de saúde do indivíduo. Ademais, a valorização e acolhimento do usuário fortalece o vínculo entre o profissional e a pessoa ${ }^{17}$.

$O$ acolhimento de enfermagem à pessoa com DM e seus familiares possibilita aproximação e confiança como forma estratégica 
de se orientar e responder possíveis questionamentos demonstrados pelo indivíduo e seus familiares que se encontram, a priori, comovidos diante do atual diagnóstico ${ }^{22}$.

Fator 5 - A promoção e aceitação da expressão de sentimentos positivos e negativos

A expressão de sentimentos positivos e negativos precisa estar presente no processo de cuidado e os cuidadores precisam assumir o papel de facilitadores $^{11}$. Na prestação de assistência de qualidade, o profissional de saúde deve conceber a pessoa com DM de forma que o estimule a exteriorizar seus sentimentos, pois é assim que será possível conhecer todos os fatores que interferem no processo saúde-doença daquele indivíduo. Medos e anseios podem estar associados ao surgimento ou agravo da patologia $^{23}$.

Lidar com o diagnóstico de diabetes desperta nas pessoas sensações como culpa, revolta e medo. Estes sentimentos precisam ser identificados e compreendidos pelos profissionais da equipe de saúde, já que essas sensações e seus desenvolvimentos poderão influenciar consideravelmente o perfil clínico e a adesão à terapêutica dos indivíduos ${ }^{19}$.

O período mais crítico para a pessoa se dá no momento do diagnóstico, e é nessa fase que o profissional de saúde deve ficar mais atento e disponível. Muitas vezes o indivíduo tende a manifestar sinais negativos relativos à doença, pois frequentemente, os profissionais de saúde focam apenas nas proibições em relação a alimentação e outros aspectos, o que de certa forma, deixa o indivíduo ainda mais aflito e descrente ${ }^{19}$.

Assim, os sentimentos negativos despertados nas pessoas frente ao diagnóstico e seu tratamento devem ser conhecidos e amenizados pelos enfermeiros. Uma pesquisa ${ }^{24}$ realizada com 20 pessoas com diabetes revelou que, quando perguntados sobre sentimentos negativos, 12 indivíduos (60\%) responderam que apresentaram sentimentos negativos em algum momento da doença. Isso demonstra que as pessoas que obtiveram pontuações mais altas, estão também em maior risco de desenvolver a depressão, leve ou grave.

O DM pode provocar sentimentos de menos-valia, inferioridade, baixa autoestima, medo, revolta, negação da doença, dentre outros fatores e o surgimento desses sentimentos está correlacionado diretamente a personalidade do indivíduo, na forma que foi dado o diagnóstico e como seus familiares e amigos reagem diante do quadro $^{25}$. Enfatiza-se também, que o emocional influencia diretamente no controle da doença, uma vez que o indivíduo pode tornar-se escravo do seu tratamento ${ }^{25}$.

Fator 6 - O uso sistemático do método científico de solução de problemas para a tomada de decisão

A promoção da pesquisa e solução sistemática de problemas é para Watson um importante fator para a ciência do cuidado. Acredita-se que sem o uso sistemático do método científico de solução de problemas, a prática eficaz é acidental na melhor das hipóteses, e é fortuita ou prejudicial na pior das hipóteses ${ }^{8}$.

O enfermeiro é visto nos âmbitos em que atua como figura de liderança e proatividade. Dessa forma, o conhecimento aprimorado fará com que o mesmo seja capaz de desenvolver estratégias que proporcionem ao cliente um bom acompanhamento, embasado em um modelo científico, assegurando uma conduta segura e efetiva ${ }^{26}$.

No entanto, o profissional de enfermagem necessita buscar conhecimentos mais aprofundados no que concerne a fisiopatologia do DM, suas comorbidades e as possíveis complicações, procurando traçar metas que estejam de acordo com a realidade da pessoa, realizando um trabalho em conjunto com uma equipe multiprofissional ${ }^{27}$.

Assim, destaca-se importante o desenvolvimento de estudos e criação de estratégias com o intuito de orientar a população a respeito do DM, que visem informar o indivíduo e seus familiares, sendo primordial trabalhar com essas pessoas o controle da doença e as possíveis complicações em caso de descontrole, os profissionais de saúde devem comprometer-se com tais ações ${ }^{28}$.

Fator 7 - A promoção do ensino-aprendizagem interpessoal

A promoção do ensino-aprendizado interpessoal inclui o processo de engajamento das pessoas envolvidas no processo de cuidado, bem como o fornecimento de informações, considerando a natureza do aprendizado e do processo interpessoal estabelecido ${ }^{8}$.

Sendo assim, é de suma importância que o profissional de enfermagem busque frequentemente o aprimoramento dos seus conhecimentos, por meio da educação continuada, permanente e outros, no intuito de proporcionar o melhor cuidado. Vale ressaltar que os profissionais que possuem 0 
conhecimento técnico-científico mais avançado precisam buscar adequar a linguagem e as informações para aqueles que o ouvem, uma vez que uma linguagem muito complexa pode-se tornar a assistência fragmentada ${ }^{29}$.

Uma das maiores contribuições do enfermeiro nesse sentido é a educação em saúde, uma vez que esta prática possibilita a promoção do ensino-aprendizagem transpessoal. A educação em saúde configura-se como uma das melhores opções para a sensibilização do usuário em relação a sua doença e por meio desta, o indivíduo com diabetes terá conhecimento em relação a patologia, como também, a hábitos que possam vir a agravá-la. A educação em saúde pode instruir a pessoa com DM sobre a importância de uma alimentação balanceada, a prática de exercícios físicos, dentre outras ações que possam vir a facilitar o controle glicêmico. É um momento que favorece a troca de informações entre o profissional enfermeiro, o usuário e o cuidador ${ }^{30}$.

Com as atividades de educação em saúde pode-se proporcionar ao indivíduo momentos de reflexão acerca da doença, do cuidado e da importância do automonitoramento para a prevenção de complicações agudas e crônicas, proporcionando uma melhor qualidade de vida ao usuário ${ }^{20}$.

A realização da educação em saúde é uma tarefa que todos os profissionais devem realizar, dentre eles o enfermeiro. No entanto, as dificuldades em relação as práticas da educação em saúde são presentes, a pessoa não reconhece esta ação como necessária para o seu tratamento, uma vez que não é algo que leva em conta medicações, horários, e indicações, ou seja, não diz respeito a uma terapia medicamentosa, baseada no modelo hospitalocêntrico. Assim, cabe ao profissional enfermeiro, como profissional capacitado para a função, desmistificar essa concepção ${ }^{31}$.

É primordial que o cliente tenha conhecimento a respeito da patologia que detém, no intuito de tomar para si, os aspectos e fatores que podem levar ao agravo, como também o controle da doença. Além do mais, o enfermeiro precisa se adequar a realidade e necessidades do cliente, uma vez que esta ação possibilita a obtenção das metas traçadas, levando em consideração que cada indivíduo possui suas particularidades ${ }^{31}$.

Cabe ao profissional de enfermagem estimular a pessoa para que este assuma uma postura proativa para a manutenção da sua saúde, vindo a desenvolver e assumindo a responsabilidade do seu autocuidado. Esse estímulo pode vir a ser realizado a partir da realização de grupos de apoio que tratem a respeito da doença, como também, com a elaboração de um plano de cuidados junto ao indivíduo ${ }^{26}$.

No cuidado realizado, deve ser estimulado também o empoderamento, cujo enfoque encontra-se na necessidade de um cuidado que envolva a participação ativa do indivíduo com DM, buscando ações resolutivas, integrativas e dialógicas. Torna-se necessário compreender e apreender o modo de viver do indivíduo e todo o seu núcleo familiar. O cuidado "empoderador", tem como intuito proporcionar à pessoa conhecimentos de si e de tudo aquilo que o rodeia $^{32}$.

Fator 8 - A promoção de um ambiente de apoio, proteção e/ou de correção mental, física, sóciocultural e espiritual

Inúmeras variáveis afetam a vida e o bem-estar da pessoa. Estas variáveis podem ser externas e internas, sendo interdependentes e afetando igualmente a saúde e a doença ${ }^{8}$. Embora Watson considere que existem inúmeras variáveis, ela enfatiza as externas neste fator de cuidado, tais como: conforto, privacidade e segurança, além de um ambiente estético e limpo $^{11}$, o que nem sempre está disponível durante assistência prestada ao cliente.

A falta de insumos interfere diretamente na qualidade de assistência de enfermagem para pessoas com DM. A limitação de recursos vai desde materiais básicos para a assistência como glicosímetros e fitas, até a infraestrutura inadequada para atendimento, o que, consequentemente, influencia diretamente na adesão ao tratamento, uma vez que o indivíduo pode sentir-se mal assistido ${ }^{33}$.

Para que a atenção às pessoas com diabetes seja realizada adequadamente são necessárias condições materiais e aporte tecnológico específico para a organização do processo de trabalho e a efetivação do cuidado ${ }^{34}$. Entretanto, há desigualdades regionais em relação a estruturas de serviços de saúde e processo de trabalho das equipes, sendo que as regiões Norte e Nordeste apresentam maior precariedade nos serviços ${ }^{35}$.

A oferta de um ambiente adequado para a prestação de assistência faz com que o usuário e o profissional se sintam mais seguros para a prestação e recebimento de cuidados. Uma assistência bem planejada permite um fluxo mais adequado e uma melhor otimização do tempo, o que é benéfico para o usuário, para a equipe e para o sistema de saúde. No entanto, muitas vezes, o profissional de enfermagem não usufrui 
de uma estrutura e insumos necessários para a realização do seu trabalho e isso pode implicar em uma descontinuidade da assistência e até mesmo, a não prestação desta. Ademais, a estrutura física não é a única responsável pela fragilidade da assistência, a falta de comunicação entre os profissionais também faz com que o acompanhamento se torne fragmentado ${ }^{23}$.

Entretanto, mesmo com tais limitações, o indivíduo com DM encontra maior apoio e cuidado, uma vez que as prioridades da atenção à saúde voltam-se para ações de promoção, proteção e recuperação das pessoas, sem exigir grandes investimentos tecnológicos ou de ponta $^{36}$. Dessa forma, há possibilidades de o indivíduo ter um acompanhamento adequado e ocorrer melhoria nos indicadores da doença.

Fator 9 - Assistência com gratificação das necessidades humanas

A assistência voltada para a gratificação das necessidades humanas procura atender aquelas necessidades do ser que são biofísicas, psicofísicas, psicossociais e intra-interpessoal ${ }^{8}$. Todas estas necessidades possuem sua importância e precisam ser analisadas para que o indivíduo se sinta satisfeito e alcance o seu desenvolvimento e a sua saúde ${ }^{11}$. A participação do ser cuidado está, principalmente, na ajuda da identificação de suas necessidades e, consequentemente, no fornecimento de subsídios para as priorizações no cuidado.

O diagnóstico de DM acarreta para o indivíduo diversas sensações que necessitam ser aceitas, compreendidas e trabalhadas pela equipe de saúde. Os profissionais precisam estar capacitados para oferecer apoio e suporte aos que necessitam, incentivando também, a expressão dos sentimentos vividos pelos indivíduos, para que estes se sintam acolhidos, confortáveis e seguros para expressarem emoções intensas e frequentemente desagradáveis ${ }^{19}$.

O cliente necessita ser concebido na sua mais íntima essência, e que sejam analisados todos os aspectos que o compõe para a obtenção de um resultado assistencial satisfatório. É necessário que haja enfoque nas necessidades, desejos e reconhecimento das potencialidades e limites do cliente. A saúde do indivíduo é dependente de seus hábitos e costumes, norteados por crenças e valores. Dessa forma, conhecer e compreender essas atitudes torna-se uma das principais ferramentas para o cuidado centrado nas necessidades e anseios do indivíduo ${ }^{32}$.
Fator 10 - A existência de fatores existenciais $e$ fenomenológicos-espirituais

A existência de fatores existenciais e fenomenológicos-espirituais faz com que o ser cuidado transcenda seu sofrimento e encontre significados na situação vivenciada. Implica no estar presente no momento de dor e de sofrimento, compreendendo as pessoas do ponto de vista de como as coisas se parecem para elas $^{11}$.

Por meio da empatia, o enfermeiro é capaz de preocupar-se verdadeiramente com o sofrimento do outro, especialmente diante das complicações do DM, tais como descontrole glicêmico, amputações de membros, dentre outros. Especificamente, as amputações causam um forte impacto e significam uma vivência difícil para a pessoa, acarretando sofrimento provocado pela dependência, pelo isolamento social e a solidão ${ }^{37}$. Dadas essas situações de sofrimento, cabe ao enfermeiro desenvolver uma relação mais próxima e humana ${ }^{9}$.

Por meio de um relacionamento horizontal na assistência de enfermagem às pessoas com DM, pode-se observar uma maior liberdade de expressão, para que os indivíduos demonstrem suas necessidades. Levar em consideração as queixas e problemas quanto à patologia remete a uma prática de atenção à saúde mais acolhedora, denotando compromisso e preocupação com o indivíduo, tornando o profissional de enfermagem uma referência, o que fortalece o vínculo entre profissional e usuário $^{17}$.

O descontrole glicêmico pode afetar diretamente a qualidade de vida do indivíduo, tornando necessário que os profissionais saibam identificar precocemente possíveis agravos e possam atuar corretamente no encaminhamento da pessoa para atenção mais complexa, com especialistas, de modo a fazer uso e integrar as redes de atenção à saúde ${ }^{26}$.

\section{Considerações finais}

Destaca-se a grande relevância da Teoria do Cuidado Humano para o norteamento das condutas adotadas pelos profissionais da área, uma vez que torna-se inviável tratar de um indivíduo, levando em consideração apenas sua patologia, sendo preciso atuar frente ao fator biopsicossocioespiritual, concebendo a pessoa como um ser possuidor de crenças, valores, desejos e anseios. 
Assim, o conhecimento fundamentado das diretrizes para assistência apontados por uma teoria de enfermagem, implica na prestação de um cuidado holístico e fortalece a interação enfermeiro-usuário com DM. A implantação dessa teoria como norteadora para as condutas de enfermagem possibilita a realização de uma assistência mais humana, empática, altruísta e diminui as sobrecargas vivenciadas pelos profissionais, estimulando-os a prestar um cuidado de qualidade.

Ressalta-se que esse estudo serve de base para o desenvolvimento de novos estudos e fomenta a prática baseada no cuidado humano e nas teorias que embasam a ciência da enfermagem, o que influencia nas relações interpessoais entre profissional, indivíduo, família, comunidade e a consequente corresponsabilidade pela saúde.

\section{Referências}

1. Flor LS, Campos MR. Prevalência de diabetes mellitus e fatores associados na população adulta brasileira. Rev. bras. epidemiol. 2017; $20 \quad$ (1): $16-29 . \quad$ DOI: https://doi.org/10.1590/19805497201700010002.

2. Sartorelli DS, Franco LJ. Tendências do diabetes mellitus no Brasil: o papel da transição nutricional. Cad. Saúde Pública. 2003; 19 Supl 1: 29-36. DOI: https://doi.org/10.1590/S0102$311 \times 2003000700004$.

3. Cortez DN, Reis, IA, Souza DAS, Macedo MML, Torres HC. Complicações e o tempo de diagnósticos na atenção primária. Acta Paul Enfermagem. 2015; 28 (3): 250-5. DOI: https://doi.org/10.1590/1982-0194201500042.

4. Santos EM, Souza VP, Correio IAG, Correio EBS. The self-care of users bearing diabetes mellitus: socio-demographic, clinical and therapeutic profiles. Fundam. care. online. 2018; 10 (3): 720-8.

5. Brasil. Ministério da Saúde. Estratégia para o cuidado da pessoa com doença crônica: diabetes mellitus. Brasília; 2013.

6. Curcio R, Lima MHM, Torres HC. Protocolo para consulta de enfermagem: assistência a pacientes com diabetes mellitus tipo 2 em insulinoterapia. Rev Gaúcha Enferm. 2009; 30 (3): 552-7.

7. Araújo ESS, Silva LF, Moreira TMM, Almeida PC, Freitas MC, Guedes MVC. Cuidado de enfermagem ao paciente com diabetes fundamentado na Teoria de King. Rev Bras Enferm [Internet]. 2018; 71 (3): 1092-8. DOI: http://dx.doi.org/10.1590/0034-7167-2016-0268
8. Watson J. Caring science as sacred science. Philadelphia: F.A. Davis; 2004.

9. Savieto RM, Leão ER. Assistência de enfermagem e Jean Watson: Uma Reflexão sobre empatia. Escola Anna Nery. 2016; 20 (1): 198202. DOI: https://doi.org/10.5935/14148145.20160026

10. Rother ET. Revisão sistemática $X$ revisão narrativa. Rev. Acta paul. Enferm, 2007; 20 (2): v-vi. DOI: https://doi.org/10.1590/S010321002007000200001

11. Silva AL, Nascimento KC, Virgilio MS, Mendonça RS. Análise dos fatores de cuidado de Watson em uma unidade de emergência. R. gaúcha Enferm. 2002; 23 (2): 27-50.

12. Baade RTW, Bueno E. Coconstrução da autonomia do cuidado da pessoa com diabetes. Interface (Botucatu). 2016; 20 (59): 941-951. DOI: https://doi.org/10.1590/1807-57622015.0130

13. Luengo CML, Mendonça ARA. Espiritualidade e qualidade de vida em pacientes com diabetes. Rev. Bioét. 2014; 22 (2): 380-7. DOI: $\quad$ https://doi.org/10.1590/198380422014222020

14. Sridhar GR. Diabetes, religion and spiritual. Int J Diabetes Dev Ctries. 2012; 33 (1): 5-7. DOI: https://doi.org/10.1007/s13410-0120097-8

15. Guimarães HP, Avezum A. O impacto da espiritualidade na saúde física. Rev. psiquiatr. clín. 2007; 34 Supl 1: 88-94. DOI: http://dx.doi.org/10.1590/s010160832007000700012

16. Kendler SK, Gardner CO, Pescott CA. Clarifying the relationship between religiosity and psychiatric illness: the impact of covariates and the specificity of buffering effects. Twin Res. 1999 Jun; 2 (2): 137-44. DOI: $10.1375 / 136905299320566004$

17. ARRUDA C, SILVA DMGV. Acolhimento e vinculo na humanização do cuidado de enfermagem às pessoas com diabetes mellitus. Rev. bras. Enferm. 2012; 65 (5): 758-766. DOI: https://doi.org/10.1590/S0034-

71672012000500007.

18. Collet N, Rozendo CA. Humanização e trabalho na enfermagem. Rev. bras. enferm. 2003; $56 \quad$ (2). DOI: https://doi.org/10.1590/S0034-

71672003000200016

19. Péres DS, Franco LJ, Santos MA. Sentimentos de mulheres após diagnóstico de diabetes tipo 2. Rev Latino-am Enfermagem. 2008; $16 \quad$ (1): 101-8. DOI: https://doi.org/10.1590/S0104$\underline{1169200800010001}$ 
20. Matsumot PM, Barreto ARB, Sakata KN, Siqueira YMC, Zoboli ELCP, Fracoli L. A educação em saúde no cuidado de usuários do programa automonitoramento glicêmico. Rev. esc. enferm. USP. 2012; 46 (3): 761-765. DOI: https://doi.org/10.1590/S008062342012000300031

21. Broca PV, Ferreira MA. Equipe de enfermagem e comunicação: contribuições para o cuidado de enfermagem. Rev Bras Enferm. 2012; $65 \quad$ (1): $\quad 97-103 . \quad$ DOI https://doi.org/10.1590/S003471672012000100014

22. Vidal AKDV, Santos JEO, Reis TS, Trindade LMP. A influência da enfermagem para minimizar o quadro de diabetes mellitus tipo 1. Revista científica Facmais. 2016; 7 (3).

23. Salci MA, Meirelles BHS, Silva DMGV. Educação em saúde para prevenção das complicações crônicas do diabetes mellitus na atenção primária. Esc Anna Nery. 2018; 22 (1): 16. DOI: https://doi.org/10.1590/2177-9465-ean2017-0262

24. Araujo KO, Andrade AN, Costa TS, Freitas MA, Nascimento MMP, Silva EN. Avaliação da qualidade de vida de portadores de diabetes mellitus tipo 2. Rev enferm UFPE on line. 2013; 7 (9): 5583-9. DOI: 10.5205/reuol.3529-29105-1SM.0709201328

25. Marcelino BD, Carvalho MDB. Reflexões sobre o diabetes tipo 1 e sua relação com o emocional. Psicologia: Reflexão e Crítica. 2005; 18 (1): 72-77.

26. Vargas CP, Lima DKC, Silva DL, Schoeller SD, Vagas MAO, Lopes SGR. Condutas dos enfermeiros da atenção primária no cuidado a pessoas com pé diabético. Rev. enferm. UFPE on line. 2017; 11 Supl 11: 4535-4545. DOI: 10.5205/reuol.11138-99362-1-

SM.1111sup201701.

27. Souza JD, Baptista MHB, Gomides DS, Pace AE. Adesão ao cuidado e diabetes mellitus nos três níveis de atenção à saúde. Esc. Anna Nery. 2017; 21 (4): 1-9. DOI: http://dx.doi.org/10.1590/2177-9465-ean-2017$\underline{0045}$.

28. Barbosa SA, Camboim FEF. Diabetes mellitus: cuidados de enfermagem para controle e prevenção de complicações. Temas em Saúde. 2016; 16 (3): 404-417.

29. Torres HC, Pace AM, Chaves FF, Melendez GV, Reis IA. Avaliação dos efeitos de um programa educativo em diabetes: ensaio clínico randomizado. Rev. Saúde Pública. 2018; 52 (8): 1-11. DOI: http://dx.doi.org/10.11606/s15188787.2018052007132.
30. Torres HC, Cortez DN, Reis IA. Avaliação da educação em grupo de diabetes na atenção primária à saúde. Cienc. enferm. 2016; 32 (3): 3545. DOI: http://dx.doi.org/10.4067/S071795532016000300035.

31. Palmeira CS, Pereira A, Melo C. Prática de enfermagem na prevenção de doenças cardiovasculares. Revista Baiana de Enfermagem. 2005; 19 (1): 83-91. DOI: http://dx.doi.org/10.18471/rbe.v19i1.3893.

32. Hammerschmidt KSA, Lenardt $\mathrm{MH}$. Tecnologia educacional inovadora para $\mathrm{O}$ empoderamento junto a idosos com diabetes mellitus. Texto contexto - enferm. 2010; 19 (2): 358-365. DOI: http://dx.doi.org/10.1590/S010407072010000200018

33. Gama CAP, Guimarães DA, Rocha GNG. Diabetes mellitus e atenção primária: percepção dos profissionais sobre os problemas relacionados ao cuidado oferecido às pessoas com diabetes. Pesquisas e Práticas Psicossociais. 2017; 12 (3): 1-16.

34. Borges DB, Lacerda JT. Ações voltadas ao controle do diabetes mellitus na atenção básica: Proposta de modelo avaliativo. Saúde debate. 2018; 42 (116): 162-178. DOI: https://doi.org/10.1590/0103-1104201811613

35. Neves RG, Duro SMS, Muniz J, Castro TRP, Faccitini LA, Tomasi E. Estrutura das unidades básicas de saúde para atenção às pessoas com diabetes: ciclo I e II do programa nacional de melhoria do acesso e qualidade. Cad. Saúde Pública. 2018; 34 (4): 1-10. DOI: https://doi.org/10.1590/0102-311x00072317

36. Silva BB, Engroff P, Sgnaolin V, Ely LS, Gomes I. Prevalência de diabetes mellitus e adesão medicamentosa em idosos da estrátegia de saúde da família de Porto Alegre/RS. Cad. saúde colet. 2016; 24 (3): 308-316. DOI: https://doi.org/10.1590/1414-

462x201600030017

37. Batista NNLAL, Luz MHBA. Vivência de pessoas com diabetes e amputação de membros. Rev. bras. enferm. 2012; 65 (2): 244-250. DOI: http://dx.doi.org/10.1590/S003471672012000200007 
Héryka Laura Calú Alves

Endereço: Rua Santa Tereza, 511, Bairro São

Miguel. Juazeiro do Norte, Ceará, Brasil.

CEP: $630105-20$

E-mail: herykalaura_@hotmail.com

Recebido em 08/11/2020

Aprovado em 01/05/2021

Publicado em 27/09/2021 